\title{
液相聚合物前驱体法制备高熵碳化物纳米粉体
}

孙娅楠 ${ }^{1}$, 叶 丽 $^{1}$, 赵文英 ${ }^{1}$, 陈凤华 ${ }^{1}$, 邱文丰 ${ }^{2}$, 韩伟健 ${ }^{1}$, 刘 伟 $^{3}$, 赵 䑣 $^{1}$ (1. 中国科学院 化学研究所, 极端环境高分子材料重点实验室, 北京 100190; 2. 华南理工大学 华南软物质科学 与技术高等研究院，广州 510641; 航天特种材料及工艺技术研究所，北京 100074)

摘 要: 高熵碳化物陶瓷是近年来发展的新型材料, 由于具有高硬度、高模量和低热导率等优异性能而备受关注。液 相聚合物前驱体法在陶瓷化过程中可以实现多元素的均匀分散，制备高摘陶瓷具有独特的优势，但是相关报道较少。 本研究以金属醇盐为原料，通过可控水解缩合反应制备了金属醇盐共聚物溶液，加入碳源烯丙基酚醛(AN)后得到了 澄清的粘稠液相高摘碳化物前驱体(PHEC), 在真空下 $1800{ }^{\circ} \mathrm{C}$ 裂解 $2 \mathrm{~h}$ 获得了 $(\mathrm{Ti}, \mathrm{Zr}, \mathrm{Hf}, \mathrm{Ta}) \mathrm{C}$ 高摘碳化物陶瓷纳米粉 末。通过不同手段对前驱体和陶瓷粉体进行表征, 结果表明: 裂解温度低于 $800{ }^{\circ} \mathrm{C}$ 所获得的样品主要为 $t-\mathrm{ZrO}_{2}$ 及氧化 物固溶体, $1000{ }^{\circ} \mathrm{C}$ 开始发生碳热还原反应形成碳化物固溶体, 温度升高至 $1800{ }^{\circ} \mathrm{C}$ 后转化为高摘碳化物陶瓷; 所得陶 瓷粉末纯度高, 元素分布均匀, 颗粒尺寸一致, 粒径 $100 \mathrm{~nm}$ 。制备的液相陶瓷前驱体具有高陶瓷产率 $(28.6 \mathrm{wt} \%$ )和低 黏度 $(150 \mathrm{mPa} \cdot)$ ) 的特点, 在极性溶剂中溶解性良好。所开发的液相前驱体法在制备高摘陶瓷纳米粉体、陶瓷纤维和陶 瓷基复合材料领域具有重要应用价值。

关 键 词: 高熵陶瓷; 碳化物; 固溶体; 纳米粉体; 液相前驱体法

中图分类号: TB383 文献标识码: A

\section{Synthesis of High-entropy Carbide Nano Powders via Liquid Polymer Precursor Route}

\author{
SUN Yanan ${ }^{1}, \mathrm{YE} \mathrm{Li}^{1}$, ZHAO Wenying ${ }^{1}$, CHEN Fenghua ${ }^{1}$, QIU Wenfeng ${ }^{2}$, \\ HAN Weijian ${ }^{1}$, LIU Wei $^{3}$, ZHAO Tong ${ }^{1}$
}

(1. Key Laboratory of Science and Technology on High-tech Polymer Materials, Institute of Chemistry, Chinese Academy of Sciences, Beijing 100190, China; 2. South China Advanced Institute for Soft Matter Science and Technology, South China University of Technology, Guangzhou 510641, China; 3. Research Institute of Aerospace Special Materials and Processing Technology, Beijing 100074, China)

\begin{abstract}
High entropy carbide ceramics have been proposed in recent years for their promising properties as high hardness, high modulus and low thermal conductivity. Liquid polymer precursor method, of which multiple elements distribute homogeneously during the pyrolysis process, is considered to be favorable in fabricating high entropy ceramics. However, few reports have studied the synthesis of liquid precursor. In this work, liquid polymer precursor of $(\mathrm{Ti}, \mathrm{Zr}, \mathrm{Hf}, \mathrm{Ta}) \mathrm{C}$ were synthesized by co-hydrolysis and polycondensation of equiatomic metal containing monomers, and adding allyl-functional novolac resin (AN) as carbon source. The obtained polymer precursors of high entropy carbide ceramics (PHECs) were soluble in propyl alcohol and stable for months. The corresponding high
\end{abstract}

收稿日期: 2020-07-13; 收到修改稿日期：2020-10-27; 网络出版日期：2020-11-15

基金项目：国家自然科学基金(21803062) National Natural Science Foundation of China (21803062)

作者简介：孙娅楠(1989-), 女，博士研究生. E-mail: sunyanan@iccas.ac.cn SUN Yanan(1989-), female, PhD candidate. E-mail: sunyanan@iccas.ac.cn

通信作者: 赵 䑣, 研究员. E-mail: tzhao@iccas.ac.cn

ZHAO Tong, professor. E-mail: tzhao@iccas.ac.cn 
entropy carbide ceramic nano powders were obtained by pyrolyzing PHEC at $1800{ }^{\circ} \mathrm{C}$ for $2 \mathrm{~h}$ in vacuum. The precursors and ceramic powders were characterized by different methods. Results reveal that the ceramic sample pyrolyzed at $800{ }^{\circ} \mathrm{C}$ are composed of $t-\mathrm{ZrO}_{2}$ and oxide solid solutions, and carbothermal reduction reaction began after pyrolyzed at $1000{ }^{\circ} \mathrm{C}$, with carbide solid solutions being generated. After being pyrolyzed at $1800{ }^{\circ} \mathrm{C}$, the samples convert to target high entropy carbide ceramics. As-obtained ceramics are characterized to be high purity with uniform distribution of nanosized particles $(\sim 100 \mathrm{~nm})$. The synthesized precursor has high ceramic yield $(28.6 \mathrm{wt} \%)$, low viscosity $(150 \mathrm{mPa} \cdot \mathrm{s})$, and good solubility in polar solvents. Therefore, the proposed liquid polymer precursor method is reliable in preparation of high entropy ceramic nano powders, ceramic fibers and ceramic matrix composites.

Key words: high entropy ceramic; carbide; solid solution; nano powder; liquid precursor

自从 2004 年高熵合金的概念被提出以来，高摘 效应给材料带来的性能改善逐渐引起人们的关注, 同时高熵概念也逐渐拓展到其他材料中，例如高熵 陶瓷材料 ${ }^{[1-5]}$ 。高熵陶瓷通常指不少于四种阳离子或 阴离子组分的单相陶瓷 ${ }^{[6]}$ 。其中高熵碳化物陶瓷的 研究主要集中在过渡金属 IVB、VB、VIB 族的碳化 物固溶体 ${ }^{[7]}$ 。这类碳化物具有很高的熔点, 且所制 备的高熵陶瓷的力学性能、隔热性能及抗氧化性能 优于单一组分, 在高温防热领域具有良好的应用前 景 ${ }^{[8-13]}$ 。目前高熵碳化物陶瓷粉体的制备方法主要 为固相法, 例如以金属碳化物粉体为原料, 经高温 和放电等离子烧结(SPS)使原子发生扩散形成单相 固溶体 ${ }^{[14-16] ;}$ 或者使用金属氧化物和石墨粉体经过 碳热还原反应制备 ${ }^{[17-18]}$; 或者采用金属单质和石墨 粉体通过室温球磨的方式, 在金属合金化的同时引 入碳到合金结构中 ${ }^{[19-20]}$ 。尽管固相法原料易得, 设备 简单, 但是制备的高摘碳化物存在以下问题: (1)固 相法需要对原料进行球磨, 以便混合得更均匀, 在 此过程中不可避免地会带来氧或其他金属杂质的污 染, 降低陶瓷的纯度, 从而对材料性能产生影响 ${ }^{[21]}$; (2)原料粉末的颗粒尺寸较大, 达到近微米级别, 不 利于原子扩散, 易造成固溶反应不完全, 无法制备

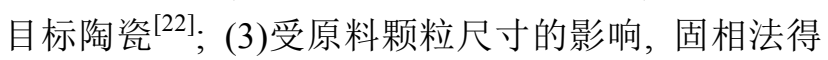
到的陶瓷粉末通常颗粒尺寸较大, 且分布不均一, 很难制备均匀的纳米陶瓷粉末 ${ }^{[23-25]}$; (4)反应原料为 固体粉末, 限制了高熵陶瓷的成型工艺性, 使其难 以制备陶瓷基复合材料或陶瓷纤维等材料。

液相聚合物前驱体法通过含金属单体聚合的方 式, 获得元素分子级别均匀分散的聚合物溶液。它 在高温陶瓷化过程中可保持各组分均匀分布, 更大 程度地促进原子迁移, 从而有利于单相固溶体的生 成 ${ }^{[26-27]}$ 。 $\mathrm{Li}$ 等 ${ }^{[28]}$ 采用过渡金属氯化物和糠醛制备了 $(\mathrm{TiZrHfNbTa}) \mathrm{C}$ 液相前驱体, 在 $2000{ }^{\circ} \mathrm{C}$ 下裂解获得
了相应的纳米陶瓷粉体，颗粒尺寸为 $(132 \pm 5) \mathrm{nm}$, 氧含量仅为 $0.22 \mathrm{wt} \%$ 。类似的, Liu 等 ${ }^{[29]}$ 采用过渡金 属氯化物通过醇解和缩聚反应制备了 $\left(\mathrm{Nb}_{1 / 3} \mathrm{Zr}_{1 / 3} \mathrm{Ta}_{1 / 3}\right) \mathrm{C}$ 前驱体, 经 $2200{ }^{\circ} \mathrm{C}$ 裂解后获得了颗粒尺寸为 $250 \mathrm{~nm}$ 的陶瓷粉体, 制备的粉体同样具有较低的氧含量 $(0.79 \mathrm{wt} \%)$, 但是存在 $8.98 \mathrm{wt} \%$ 的富余碳, 并且粉体 颗粒也存在一定程度的烧结。这些研究结果展现出 液相聚合物前驱体法制备高熵碳化物陶瓷纳米粉体 的优势 ${ }^{[30]}$ 。但是上述研究均采用金属氯化物作为原 料, 与醇类溶剂形成金属烷氧基化合物, 其中活泼 的金属烷氧键极易发生水解, 这不仅会影响前驱体 的稳定性及加工工艺性, 而且会破坏金属元素的分 子级均匀分散, 从而影响陶瓷元素的均匀性; 而且 前驱体中所含的 $\mathrm{Cl}^{-}$在陶瓷化过程中会对设备或纤 维基体造成腐蚀。加工性能良好、对设备低腐蚀的 高熵碳化物陶瓷前驱体还未有报道。

本工作提出一种制备高熵碳化物陶瓷前驱体的 方法。以金属醇盐为原料, 通过可控水解缩合反应 制备含有 $\mathrm{Ti} 、 \mathrm{Zr} 、 \mathrm{Hf} 、 \mathrm{Ta}$ 的聚合物溶液, 加入烯丙 基酚醛作为碳源, 得到澄清的液相前驱体 PHEC, 再经高温裂解获得相应的高熵碳化物陶瓷纳米粉 末。对 PHEC 的稳定性和陶瓷化过程进行了研究, 并对陶瓷粉体的晶体结构和微观形貌进行表征。

\section{1 实验方法}

\section{1 前驱体的制备}

钛醇盐和锆醇盐: 钛酸丙酯 $\left(\mathrm{Ti}\left(\mathrm{OC}_{3} \mathrm{H}_{7}\right)_{4}\right.$, 纯度 $>99 \mathrm{wt} \%)$, 锆酸丙酯 $\left(\mathrm{Zr}\left(\mathrm{OC}_{3} \mathrm{H}_{7}\right)_{4}\right.$, 纯度 $>72 \mathrm{wt} \%$, 正 丙醇溶液), 山东和瑞东精细化学有限公司生产; 铪 醇盐和钽醇盐按文献[31]的方法制备; 碳源为烯丙 基酚醛(实验室自制)。首先在室温下将 $0.2 \mathrm{~mol}$ 的四 种金属醇盐分别加入 $0.2 \mathrm{~mol}$ 的乙酰丙酮, 共混后 
回流 $2 \mathrm{~h}$; 然后滴入 $0.8 \mathrm{~mol}$ 的水, 继续回流 $1 \mathrm{~h}$ 后减 压除去大部分溶剂, 向体系内加入烯丙基酚醛, 摚 拌均匀后得到均一澄清的液相前驱体溶液 PHEC。

\subsection{PHEC 的固化及陶瓷化}

将 PHEC 置于烘箱中进行固化, 固化程序为 160 、 180、200、220、250 ${ }^{\circ} \mathrm{C}$ 各 $1 \mathrm{~h}$, 升温速率为 $3{ }^{\circ} \mathrm{C} / \mathrm{min}$ 。

将 PHEC 固化所得的样品置于石墨炉中, 在 $600 \sim 1800{ }^{\circ} \mathrm{C}$ 真空条件下进行裂解, 保温 $2 \mathrm{~h}$, 得到 相应温度下的裂解产物。

\section{3 表征}

采用上海平轩科学仪器有限公司 NDJ-79 型旋 转黏度计, 参照 GJB1059.2-90 在 $25{ }^{\circ} \mathrm{C}$ 测试 PHEC 的黏度, 室温储存 $60 \mathrm{~d}$ 后重复测试。采用德国布鲁 克公司生产的 Bruker Tensor 27 型红外分析仪在室 温下对样品进行红外分析, 采用溴化钾盐片涂膜法 制备液体样品。采用德国布鲁克公司生产的 Bruker Advance III HD 400 核磁共振谱仪, 以 $\mathrm{CDCl}_{3}$ 为溶 剂, TMS 为内标, 在室温下测定 PHEC 的核磁谱图。 采用德国 NETZSCH 公司的 STA449F3 型同步热分 析仪对样品进行热失重分析, 以 $\mathrm{N}_{2}$ 为测试氛围, 升 温速率为 $10{ }^{\circ} \mathrm{C} / \mathrm{min}$, 测试范围为室温 $(\mathrm{RT}) \sim 1450{ }^{\circ} \mathrm{C}$ 。 采用日本 Rigaku D/max $2500 \mathrm{X}$ 射线衍射仪进行结 构分析, 辐射源为 $\mathrm{CuK} \alpha(\lambda=0.154060 \mathrm{~nm})$, 扫描范围 为 $10^{\circ} \sim 80^{\circ}$, 扫描速率为 $8\left(^{\circ}\right) / \mathrm{min}$ 。采用美国 Thermal 公司的 IRIS Intrepid II 型电感耦合等离子光谱仪 (ICP-OES)测试样品的 $\mathrm{Ti} 、 \mathrm{Zr} 、 \mathrm{Hf} 、 \mathrm{Ta}$ 元素含量。 采用日本 Hitachi S4800 型扫描电子显微镜观察样品 表面微观形貌，并用所配置的 EDX 附件进行元素分 析。采用日本 JEOL JEM-2100F 透射电镜在 $200 \mathrm{kV}$ 加速电压下观察样品, 采用软件 JADE 对不同样品 进行晶粒尺寸和晶体结构分析。

\section{2 结果与讨论}

\subsection{PHEC 的物性表征}

制备的前驱体 PHEC 外观呈现为均一的红棕色 液体(如图 1 所示), 在正丙醇等极性溶剂中具有良 好的溶解性。从表 1 中可以看到在 $25{ }^{\circ} \mathrm{C}$ 下通过旋 转粘度计测得的 PHEC 黏度为 $150 \mathrm{mPa} \cdot \mathrm{s}$, 室温储存 $60 \mathrm{~d}$ 后黏度仅增加 $8 \%$; 前驱体的总金属含量为 $28 \mathrm{wt} \%$, 在 $1800{ }^{\circ} \mathrm{C}$ 的陶瓷产率为 $28.6 \mathrm{wt} \%$ 。 PHEC 具有室温黏度低和储存稳定性良好的特点, 有望应 用于前驱体浸渍裂解(PIP)法制备高熵碳化物陶瓷 基复合材料，同时较高的金属含量及陶瓷产率可以 大大提高浸渍效率。

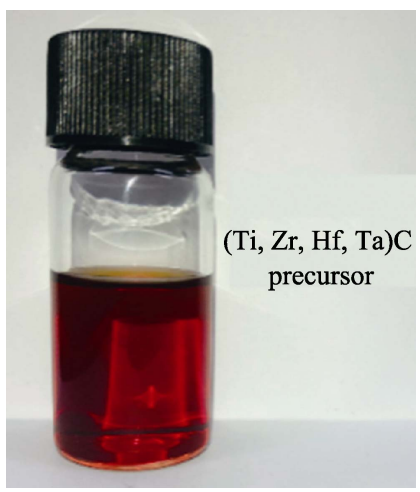

图 1 PHEC 的外观图片

Fig. 1 Photograph of PHEC

表 1 PHEC 的金属含量及陶瓷产率及粘度

Table 1 Metal content, yield and viscosity of PHEC

\begin{tabular}{|c|c|c|c|c|}
\hline \multirow{2}{*}{ Item } & \multirow{2}{*}{$\begin{array}{c}\text { Metal } \\
\text { content/wt\% }\end{array}$} & \multirow{2}{*}{$\begin{array}{l}\text { Yield/ } \\
\mathrm{wt} \%\end{array}$} & \multicolumn{2}{|c|}{ Viscosity $/(\mathrm{mPa} \cdot \mathrm{s})$} \\
\hline & & & 1 & 2 \\
\hline$(\mathrm{Ti}, \mathrm{Zr}, \mathrm{Hf}, \mathrm{Ta}) \mathrm{C}$ & 28 & 28.6 & 150 & 162 \\
\hline
\end{tabular}

1: The viscosity of PHEC was measured as synthesized; 2 : The viscosity of PHEC was measured after stored at room temperature for $60 \mathrm{~d}$

\subsection{PHEC 的结构表征}

制备的 PHEC 特征基团通过 FT-IR 进行表征, 结果如图 2 所示。位于 $3443 \mathrm{~cm}^{-1}$ 处的吸收峰对应 着基吸收峰，由于经历过水解反应，制备的 PHEC 羟基含量较少。位于 1593、1518 和 $1275 \mathrm{~cm}^{-1}$ 处的 吸收峰分别对应乙酰丙酮 $(\mathrm{Hacac}) \mathrm{C}=\mathrm{O} 、 \mathrm{C}=\mathrm{C}$ 和 $\mathrm{C}-\mathrm{CH}_{3}$ 的吸收峰, 相较于乙酰丙酮的 $\mathrm{C}=\mathrm{O}$ 和烯烃中 的 $\mathrm{C}=\mathrm{C}$, 二者均发生了红移, 说明乙酰丙酮已经与 过渡金属形成了配位结构, 其中 $3082 \mathrm{~cm}^{-1}$ 处的微 弱吸收峰对应碳源烯丙基酚醛的末端双键氢伸缩振 动。而位于 $661 、 442 \mathrm{~cm}^{-1}$ 处的吸收峰分别对应 Ti-O 和 $\mathrm{Hf}-\mathrm{O}$ 的吸收峰; $\mathrm{Zr}-\mathrm{O}$ 和 $\mathrm{Ta}-\mathrm{O}$ 的吸收峰较接近, 出现在 $543 \mathrm{~cm}^{-1}$ 处。进一步通过 ${ }^{1} \mathrm{H}$ NMR 对 PHEC 的结构进行了表征, 结果如图 3 所示。 $\delta=5.5$ 和 2.0 处峰对应乙酰丙酮的烯醇式的 $-\mathrm{CH}$ 和 $\mathrm{CH}_{3} ; \delta=3.6$ 和 1.6 处峰分别对应溶剂正丙醇的 $-\mathrm{CH}_{2}$ 结构, $\delta=1.4$

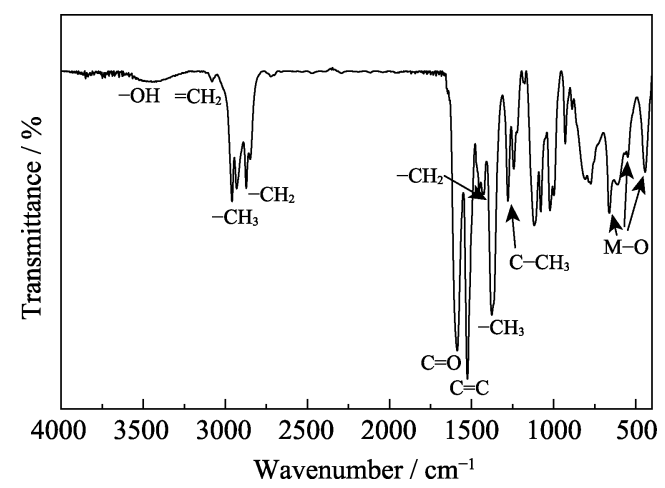

图 2 PHEC 的红外结构图谱

Fig. 2 FT-IR spectrum of PHEC 


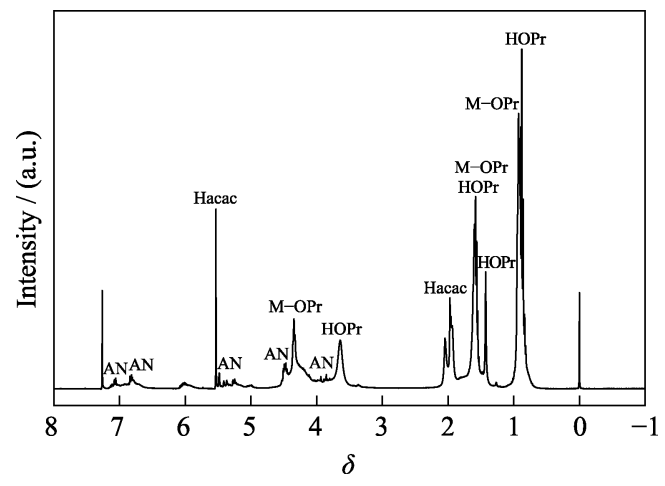

图 3 PHEC 的 ${ }^{1} \mathrm{H}$ NMR 图谱

Fig. $3{ }^{1} \mathrm{H}$ NMR spectra of PHEC

和 0.9 处峰分别对应正丙醇的 $-\mathrm{OH}$ 及 $\mathrm{CH}_{3}$ 结构, 其中 $\delta=1.6$ 和 0.9 处峰与金属-丙氧基(M-OPr)重合; $\delta=4.3$ 处 的宽峰对应金属-丙氧基(M-OPr)的结构。聚合物 AN 由于化学环境复杂, ${ }^{1} \mathrm{HNMR}$ 图中呈现多处的宽峰。

\subsection{PHEC 的陶瓷化过程}

通过热失重分析对 PHEC 固化物的裂解行为进 行了研究, 如图 4 所示。由图可看出, PHEC 固化物 在室温到 $1450{ }^{\circ} \mathrm{C}$ 范围主要有四个阶段的失重: (1) RT $250{ }^{\circ} \mathrm{C}$ 阶段, 样品失重约 $5 \mathrm{wt} \%$, 这主要是 由于固化样品吸收的水汽挥发及样品中残留的羟基 脱除所造成的; (2) 250 500 ${ }^{\circ} \mathrm{C}$ 介介段, 样品失重明显, 达到 $20 \mathrm{wt} \%$ 左右, 在此温度范围内, 前驱体的有机 骨架开始裂解, 大量金属-有机基团键断裂, 样品发 生有机一无机转化 ${ }^{[32]}$; (3) 500 900 ${ }^{\circ} \mathrm{C}$ 阶段, 失重较 小, 约为 $6 \mathrm{wt} \%$, 主要是前驱体进一步裂解成为无机 物所致; (4) 900 1450 ${ }^{\circ} \mathrm{C}$ 阶段, 样品迅速失重 16wt\% 左右，这主要是由于发生了金属氧化物和碳之间的 碳热还原反应。

为了研究 PHEC 陶瓷化过程的晶体结构演变, 对不同温度裂解的产物进行了 XRD 表征, 如图 5 所 示。由图 5(A)可看出, $600{ }^{\circ} \mathrm{C}$ 裂解产物的衍射谱呈 现较宽的馒头峰, 说明样品为非晶态。热处理温度 为 $800{ }^{\circ} \mathrm{C}$, 产物开始出现 $t-\mathrm{ZrO}_{2} 、(\mathrm{Ti}, \mathrm{Zr}) \mathrm{O}_{x} 、(\mathrm{Ti}, \mathrm{Hf}) \mathrm{O}_{x}$

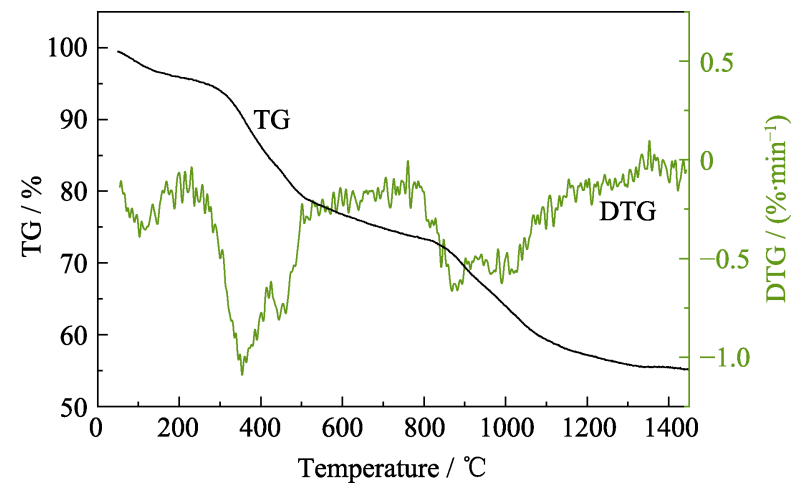

图 4 PHEC 固化样品的热失重曲线

Fig. 4 TGA curves of cured PHEC
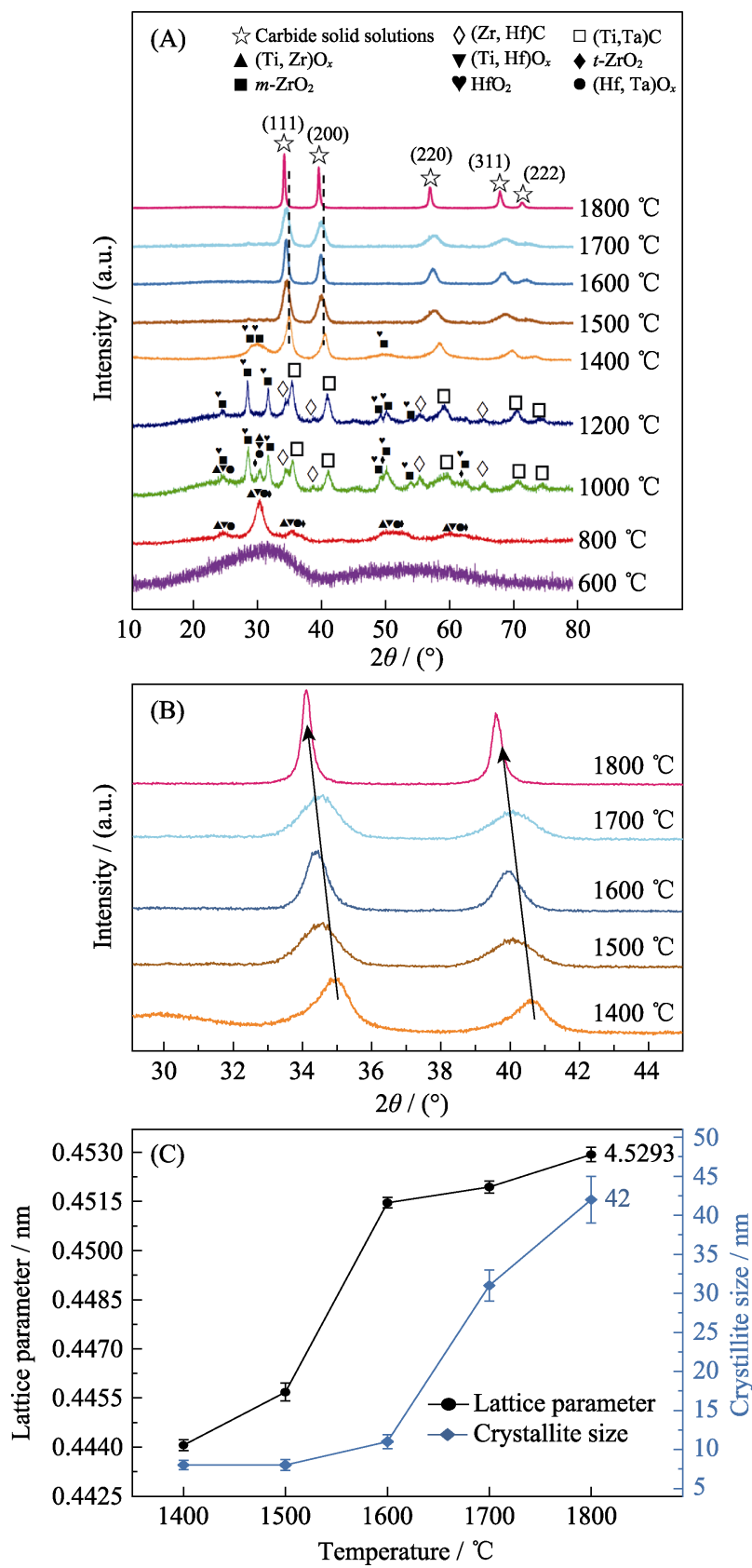

图 5 PHEC 裂解过程的 XRD 表征

Fig. 5 Characterization of pyrolysis process for PHEC

(A) XRD patterns of products pyrolyzed at different temperatures; (B) Enlarged images of XRD patterns of products pyrolyzed at 1400 $1800{ }^{\circ} \mathrm{C}$; (C) Lattice parameter and crystallite size of samples pyrolyzed at $1400-1800{ }^{\circ} \mathrm{C}$

和( $\mathrm{Hf}, \mathrm{Ta}) \mathrm{O}_{x}$ 等氧化物固溶体。 $1000{ }^{\circ} \mathrm{C}$ 裂解产物中 $t-\mathrm{ZrO}_{2}$ 及几种氧化物固溶体的衍射峰变弱, 同时观 察到(Zr, Hf)C 和(Ti，Ta)C 碳化物固溶体的衍射峰, 表明发生了碳热还原反应。1200 ${ }^{\circ} \mathrm{C}$ 裂解后, $(\mathrm{Zr}$, $\mathrm{Hf}) \mathrm{C}$ 和( $\mathrm{Ti}, \mathrm{Ta}) \mathrm{C}$ 两种碳化物固溶体的衍射峰逐渐增 强, 并呈现靠拢的趋势, 表明固溶反应在不断进行, 但是仍然存在大量的氧化物 $\mathrm{HfO}_{2}$ 和 $m-\mathrm{ZrO}_{2} \circ 1400{ }^{\circ} \mathrm{C}$ 裂解产物中氧化物的衍射峰减小, 碳化物固溶体的 衍射峰开始增强, 呈现为较宽的单峰; 残留的 $\mathrm{HfO}_{2}$ 
和 $m-\mathrm{ZrO}_{2}$ 的衍射峰表明该温度下碳热还原反应还 未进行完全。

裂解温度为 $1500{ }^{\circ} \mathrm{C}$ 时, 几乎观察不到氧化物 的衍射峰，表明碳热还原反应基本完成; 碳化物固 溶体的衍射峰形较宽, 表明其晶粒尺寸较小, 但是 由于固溶反应未进行完全, 所以衍射峰形状不对 称。从图 5(B) 1400 1800 ${ }^{\circ} \mathrm{C}$ 裂解产物的局部 XRD 放大图可以看出，衍射峰逐渐变得尖锐，并且向左 偏移。这是由于低温时碳热还原反应未进行完全, 在更高的裂解温度下，碳热还原反应仍在进行，晶 格中溶解的氧不断被碳置换, 增大了陶瓷晶体的晶 面间距，通过布拉格方程 $n \lambda=2 d \sin \theta$ 可知，晶面间距 增大会引起衍射角减小, 从而导致衍射峰向左移 动。 $1800{ }^{\circ} \mathrm{C}$ 裂解产物的衍射峰峰形对称, 表现为特 征的面心立方晶体结构(FCC)。

由图 5(A)的 XRD 图谱进一步计算得到 1400 $1800{ }^{\circ} \mathrm{C}$ 裂解产物的的晶胞参数和晶粒尺寸变化图, 如图 5(C)所示。可以看出, 随着裂解温度的升高, 固 溶反应的进行程度不断增强, 晶胞参数呈现增加的 趋势, 说明碳化物固溶体晶体结构在高温作用下不 断完善; 晶粒也不断长大, 由 $8 \mathrm{~nm}$ 增大至 $42 \mathrm{~nm}$ 。 $1800{ }^{\circ} \mathrm{C}$ 的陶瓷化样品计算所得到的晶胞参数为 $0.45293 \mathrm{~nm}$, 同维加德定律计算值 $(0.45282 \mathrm{~nm})$ 相近, 说明在此温度下已经形成了 ( Ti, Zr, Hf, Ta)C 高熵碳 化物固溶体。由此推测 PHEC 的裂解过程如图 6 所示。

通过元素分析结果，计算得到 $n(\mathrm{Ti}): n(\mathrm{Zr})$ : $n(\mathrm{Hf}): n(\mathrm{Ta})=1.00: 1.02: 1.04: 1.05$, 与投料比接近。 与固相法合成的 $(\mathrm{Ti}, \mathrm{Zr}, \mathrm{Hf}, \mathrm{Ta}) \mathrm{C}$ 碳化物固溶体中存 在氧化物和 $\mathrm{ZrC}$ 残余的情况相比 ${ }^{[22]}$, 液相前驱体法 制备的陶瓷粉末具有高纯度的特点。通过上述晶体 结构分析可知, 液相前驱体中各元素在树脂中处于

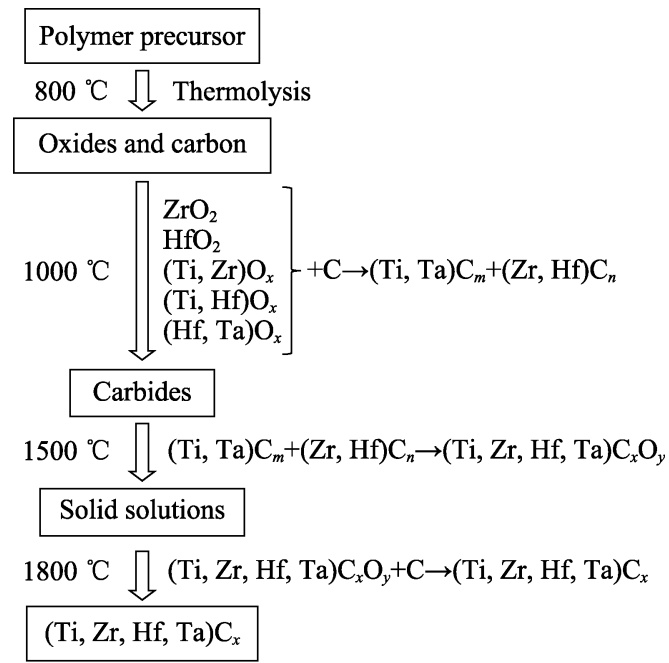

图 6 PHEC 的裂解过程原理示意图及可能发生的化学反应 Fig. 6 Schematic diagram of pyrolysis process for PHEC and possible reactions
分子级别的分散，在高温陶瓷化过程中各元素均匀 分散，因此碳热还原反应进行得较为充分，从而避 免了氧化物的残留; 同时元素的均匀分散缩短了原子 的迁移距离，有利于金属原子充分扩散得到目标高 熵碳化物陶瓷，避免了某些元素组分的扩散不完全。

对 PHEC $1800{ }^{\circ} \mathrm{C}$ 的裂解产物进行了微观形貌 表征。由图 7(A)的 SEM 照片可以看出, 样品颗粒分 布均匀，尺寸约为 $100 \mathrm{~nm}$, 颗粒之间没有明显的烧 结。对选定区域的 Ti、 $\mathrm{Zr} 、 \mathrm{Hf} 、 \mathrm{Ta}$ 元素的面分布进 行了分析, 如图 7(B)所示, 陶瓷粉末表面各金属元 素分布均匀。采用透射电镜观察 PHEC $1800{ }^{\circ} \mathrm{C}$ 裂 解产物的微观结构。如图 8(A)所示, 陶瓷粉末的颗 粒均匀, 分散性好, 无明显团聚现象; 颗粒尺寸约 为 $100 \mathrm{~nm}$, 与 SEM 观察结果一致。从图 8(B)的高 倍 HRTEM 照片可以看到, 碳化锆晶体外部包裹着 排列较规整的石墨碳，厚度约为 $2 \mathrm{~nm}$; 晶体内部可 以观察到有序排列的晶格条纹，间距为 $0.260 \mathrm{~nm}$, 对应( $\mathrm{Ti}, \mathrm{Zr}, \mathrm{Hf}, \mathrm{Ta}) \mathrm{C}$ 晶体 $\mathrm{FCC}$ 结构的(111)晶面，与 XRD 的计算结果 $0.260 \mathrm{~nm}$ 一致。图 8(C) 为图 8(A) 的元素面分布图, 可以看出 $\mathrm{Ti} 、 \mathrm{Zr} 、 \mathrm{Hf} 、 \mathrm{Ta}$ 四种元 素分布均匀, 无元素偏析或富集的情况。综合 XRD、 SEM 及 TEM 的表征结果, 可以证明获得了元素组 成均一、颗粒尺寸 $100 \mathrm{~nm}$ 的 $(\mathrm{Ti}, \mathrm{Zr}, \mathrm{Hf}, \mathrm{Ta}) \mathrm{C}$ 高熵 碳化物纳米陶瓷粉末。

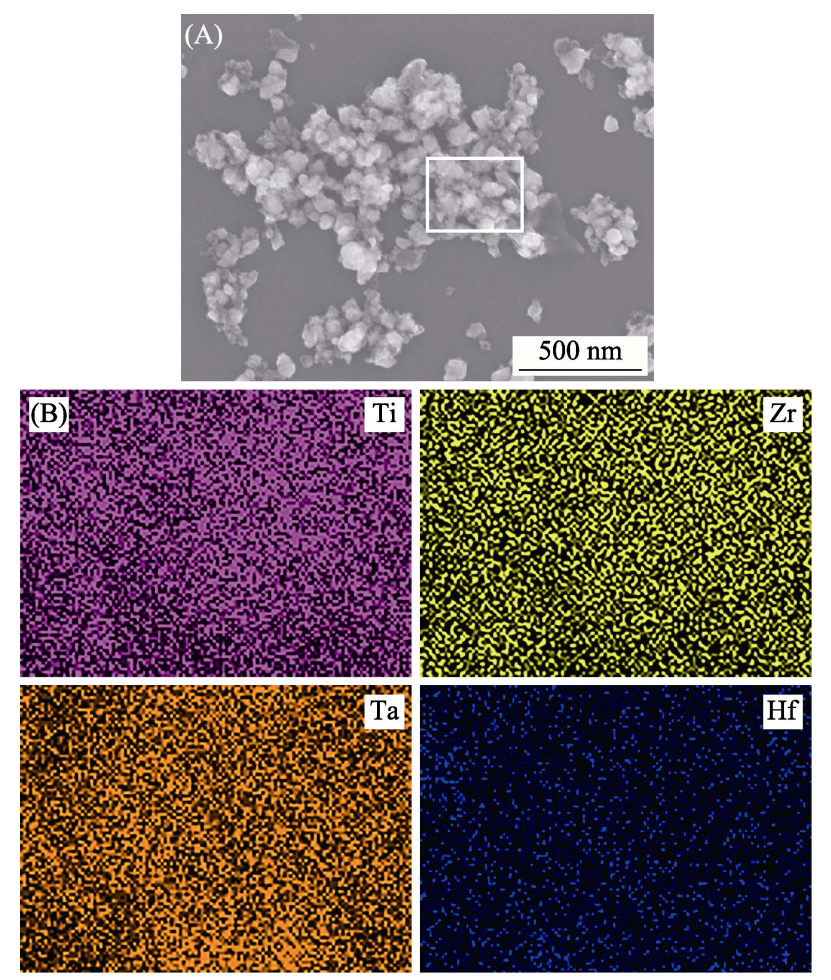

图 $71800{ }^{\circ} \mathrm{C}$ 裂解 PHEC 陶瓷粉末的 SEM 表征

Fig. 7 SEM analyses of PHEC ceramic powders pyrolyzed at $1800{ }^{\circ} \mathrm{C}$

(A) SEM image; (B) EDS mapping of selected pattern marked with rectangle 

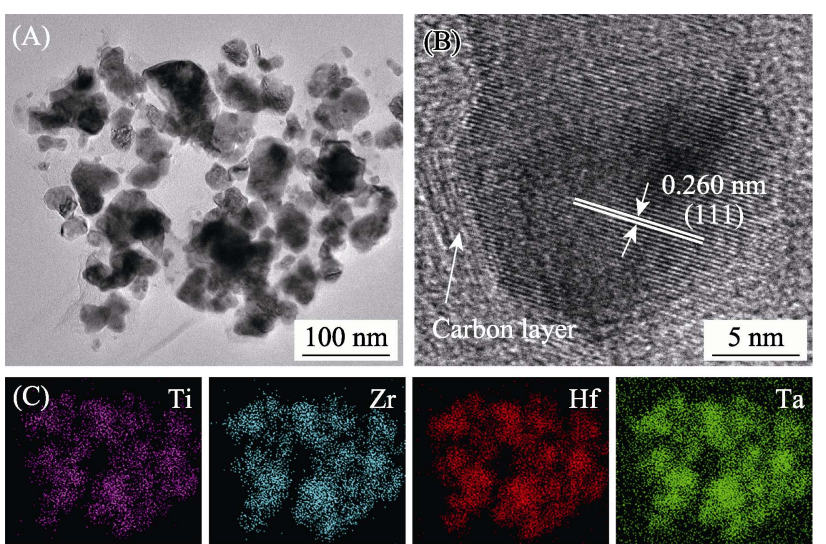

图 8 PHEC $1800{ }^{\circ} \mathrm{C}$ 裂解陶瓷粉末的 TEM 分析

Fig. 8 TEM analyses of PHEC ceramic powders pyrolyzed at $1800{ }^{\circ} \mathrm{C}$

(A) TEM image; (B) HR-TEM image; (C) EDS mapping of Ti, Zr, Hf and Ta

\section{3 结论}

采用金属醇盐可控水解聚合的方法制备了 ( Ti, $\mathrm{Zr}, \mathrm{Hf}, \mathrm{Ta}) \mathrm{C}$ 液相聚合物前驱体，经 $1800{ }^{\circ} \mathrm{C}$ 陶瓷化后 得到相应的高熵碳化物纳米陶瓷粉体。制备的粉体 颗粒尺寸为 $100 \mathrm{~nm}$, 分布均匀, 颗粒间几乎无烧结 行为; 具有 FCC 晶体结构, 元素分布均匀, 无富集或 偏析。所合成的前驱体具有高金属含量的特点 (28wt\%), 室温储存 $60 \mathrm{~d}$ 后仍然保持澄清均一的状态, 粘度仅增加 $8 \%$, 且无 $\mathrm{Cl}^{-}$, 对设备损伤小。本研究制 备的高熵碳化物纳米陶瓷前驱体, 为高熵碳化物陶 瓷纤维及复合材料的应用提供了良好的材料。

\section{参考文献:}

[1] YEH JIEN-WEI, CHEN SWE-KAI, LIN SU-JIEN, et al. Nanostructured high-entropy alloys with multiple principal elements:novel alloydesign concepts andoutcomes. Advanced Engineering Materials, 2004, 6: 299-303.

[2] CANTOR B, CHANG ITH, KNIGHT P, et al. Microstructural development in equiatomic multicomponent alloys. Materials Science and Engineering: A, 2004, 375-377: 213-218.

[3] CHEN LEI, WANG KAI, SU WEN-TAO, et al. Research progress of transition metal non-oxide high-entropy ceramics. Journal of Inorganic Materials, 2020, 35(7): 748-758.

[4] OSES C, TOHER C, CURTAROLO S. High-entropy ceramics. Nature Reviews Materials, 2020, 5(4): 295-309.

[5] GILD J, ZHANG YUAN-YAO, HARRINGTON T, et al. Highentropy metal diborides: a new class of high-entropy materials and a new type of ultrahigh temperature ceramics. Scientific Reports, 2016, 6(1): 37946.

[6] ZHANG RUI-ZHI, REECE M J. Review of high entropy ceramics: design, synthesis, structure and properties. Journal of Materials Chemistry A, 2019, 7(39): 22148-22162.

[7] SARKER P, HARRINGTON T, TOHER C, et al. High-entropy high-hardness metal carbides discovered by entropy descriptors. Nature Communications, 2018, 9(1): 4980 .

[8] 顾俊峰, 邹冀, 张帆, 等. 高熵陶瓷材料研究进展. 中国材料进 展, 2019, 38(9): 855-886.

[9] YE BEI-LIN, WEN TONG-QI, CHU YAN-HUI. High-temperature oxidation behavior of $\left(\mathrm{Hf}_{0.2} \mathrm{Zr}_{0.2} \mathrm{Ta}_{0.2} \mathrm{Nb}_{0.2} \mathrm{Ti}_{0.2}\right) \mathrm{C}$ high-entropy ceramics in air. Journal of the American Ceramic Society, 2019, 103(1): 500-507.

[10] YAN XUE-LIANG, CONSTANTIN L, LU YONG-FENG, et al. $\left(\mathrm{Hf}_{0.2} \mathrm{Zr}_{0.2} \mathrm{Ta}_{0.2} \mathrm{Nb}_{0.2} \mathrm{Ti}_{0.2}\right) \mathrm{C}$ high-entropy ceramics with low thermal conductivity. Journal of the American Ceramic Society, 2018,
101(10): 4486-4491.

[11] ROST C M, BORMAN T, HOSSAIN M D, et al. Electron and phonon thermal conductivity in high entropy carbides with variable carbon content. Acta Materialia, 2020, 196: 231-239.

[12] CHEN HENG, XIANG HUI-MIN, DAI FU-ZHI et al. High porosity and low thermal conductivity high entropy $\left(\mathrm{Zr}_{02} \mathrm{Hf}_{0.2} \mathrm{Ti}_{0.2} \mathrm{Nb}_{0.2} \mathrm{Ta}_{0.2}\right)$ C. Journal of Materials Science \& Technology, 2019, 35(8): 1700-1705.

[13] CSANÁDI T, VOJTKO M, DANKHÁZI Z, et al. Small scale fracture and strength of high-entropy carbide grains during microcantilever bending experiments. Journal of the European Ceramic Society, 2020, 40(14): 4774-4782.

[14] WEI XIAO-FENG, LIU JI-XUAN, LI FEI, et al. High entropy carbide ceramics from different starting materials. Journal of the European Ceramic Society, 2019, 39(10): 2989-2994.

[15] PENG CHONG, GAO XIANG WANG MING-ZHI, et al. Diffusioncontrolled alloying of single-phase multi-principal transition metal carbides with high toughness and low thermal diffusivity. Applied Physics Letters, 2019, 114(1): 011905-1-5.

[16] MOSKOVSKIKH D O, VOROTILO S, SEDEGOV A S, et al. Highentropy (HfTaTiNbZr)C and (HfTaTiNbMo)C carbides fabricated through reactive high-energy ball milling and spark plasma sintering. Ceramics International, 2020, 46(11): 19008-19014.

[17] SURE J, SRI MAHA VISHNU D, KIM H K, et al. Facile electrochemical synthesis of nanoscale (TiNbTaZrHf)C high-entropy carbide powder. Angewandte Chemie International Edition, 2020, 59(29): 11830-11835.

[18] WANG KAI, CHEN LEI, XU CHEN-GUANG et al. Microstructure and mechanical properties of (TiZrNbTaMo)C high-entropy ceramic. Journal of Materials Science \& Technology, 2020, 39: 99-105.

[19] CHICARDI E, GARCÍA-GARRIDO C, GOTOR F J. Low temperature synthesis of an equiatomic (TiZrHfVNb) $\mathrm{C}_{5}$ high entropy carbide by a mechanically-induced carbon diffusion route. Ceramics International, 2019, 45(17): 21858-21863.

[20] CHICARDI E, GARCIIA-GARRIDO C, HERNÁNDEZ-SAZ J, et al. Synthesis of all equiatomic five-transition metals high entropy carbides of the IVB (Ti, Zr, Hf) and VB (V, Nb, Ta) groups by a low temperature route. Ceramics International, 2020, 46(13): 21421-21430.

[21] GILD J, KAUFMANN K, VECCHIO K, et al. Reactive flash spark plasma sintering of high-entropy ultrahigh temperature ceramics. Scripta Materialia, 2019, 170: 106-110.

[22] CASTLE E, CSANADI T, GRASSO S, et al. Processing and properties of high-entropy ultra-high temperature carbides. Scientific Reports, 2018, 8(1): 8609.

[23] FENG LUN, FAHRENHOLTZ W G, HILMAS G E, et al. Synthesis of single-phase high-entropy carbide powders. Scripta Materialia, 2019, 162: 90-93.

[24] YE BEI-LIN, NING SHAN-SHAN, LIU DA, et al. One-step synthesis of coral-like high-entropy metal carbide powders. Journal of the American Ceramic Society, 2019, 102(10): 6372-6378.

[25] ZHOU JIE-YANG, ZHANG JIN-YONG, ZHANG FAN, et al. High-entropy carbide: a novel class of multicomponent ceramics. Ceramics International, 2018, 44(17): 22014-22018.

[26] LU YAN, SUN YA-NAN, ZHANG TU-ZI, et al. Polymer-derived $\mathrm{Ta}_{4} \mathrm{HfC}_{5}$ nanoscale ultrahigh-temperature ceramics: synthesis, microstructure and properties. Journal of the European Ceramic Society, 2019, 39(2/3): 205-211.

[27] SUN YA-NAN, YANG CHUN-MING, LU YAN, et al. Transformation of metallic polymer precursor into nanosized $\mathrm{HfTaC}_{2}$ ceramics. Ceramics International, 2020, 46(5): 6022-6028.

[28] LI FEI, LU YING, WANG XIN-GANG, et al. Liquid precursorderived high-entropy carbide nanopowders. Ceramics International, 2019, 45(17): 22437-22441.

[29] LIU HONG-HUA, DU BIN, CHU YAN-HUI. Synthesis of the ternary metal carbide solid-solution ceramics by polymer-derivedceramic route. Journal of the American Ceramic Society, 2020, 103(5): 2970-2974.

[30] DU BIN, LIU HONG-HUA, CHU YAN-HUI. Fabrication and characterization of polymer-derived high-entropy carbide ceramic powders. Journal of the American Ceramic Society, 2020, 103(8): 4063-4068.

[31] LU YAN, YE LI, HAN WEI-JIAN, et al. Synthesis, characterization and microstructure of tantalum carbide-based ceramics by liquid polymeric precursor method. Ceramics International, 2015, 41(9): $12475-12479$

[32] LIU DAN, CAI TAO, QIU WEN-FENG, et al. Synthesis, characterization, and microstructure of $\mathrm{ZrC} / \mathrm{SiC}$ composite ceramics via liquid precursor conversion method. Journal of the American Ceramic Society, 2014, 97(4): 1242-1247. 